\title{
Scalable Automation System Based on Raspberry
}

\author{
Chadwick Carreto, Blanca Carvajal, Alejandro Diaz \\ Instituto Politecnico Nacional, Escuela Superior de Cómputo, Mexico City, \\ Mexico \\ carretoa@ipn.mx,bcarvjal@ipn.mx, adiazc@ipn.mx
}

\begin{abstract}
This work consists of a scalable automation system divided into two parts; the first is the handling of variables such as lighting, air conditioning, curtains, etc. in hospitals or care for the elderly that allows the user to add, configure and control various electronic devices via a graphical interface or wired remote so you can tailor the environment to their needs. The second part consists of the constant monitoring of the variables temperature and heart rate of older people through the application; in order to send an alert to medical personnel in the area if these variables are outside the set range.
\end{abstract}

Keywords: Domotic, monitoring, raspberry, automatization.

\section{Introduction}

Currently the advances that have been in technology, this has been used to facilitate human life as we know have been applied in various areas such as education, medicine, transportation and entertainment among others. "The technology applied to home", known as Home Automation, integrating automation and information technology to improve the comfort, safety and welfare of households. However, the automation does not apply only in dwellings, also you can be applied in buildings and hospitals, by some-we mention places.

Hospitals and care centers aim to always buy the best technology both manage to offer patients an environment comfortable and pleasant to meet your needs, without neglecting the medical care they require; so it is necessary to monitor people to know their status at any time without the attendant go to the room can use that time in patients who need urgent medical attention.

Commercially there are several companies in the market that offer automation products. However, it was observed that hospital automation systems currently focus on patient monitoring and automation environment; but do not cover all areas; i.e., only specialize in either monitoring or automation. So our system aims to combine control of some variables such as light, temperature, curtains, among other environments (rooms); as well as patient monitoring and alerting medical personnel if there is a problem.

On the other hand, existing home automation systems are static systems, i.e., are programmed to fit the user, but once programmed cannot easily change, so if the user changes a system component, this must be reprogrammed, process often expensive 
because it requires the programmer to go to the location to make changes in programming (within the device).

Therefore, the developed system provides the user the ease of adding new devices to the system and display an alert if there is a serious problem in some areas; for example, if there is smoke, an audible alert is activated and the user is notified by the application that is being detected smoke in certain area.

Another advantage over existing systems is the possibility to add more microcomputers to increase system capacity, thus if the place where the system is installed it after a while only to be acquired, connect and configure expands devices to work with those already installed previously.

The main beneficiaries of our system will be both medical institutions such as hospitals, retirement homes, places for special care, etc. and homes where required or desired to maintain control and / or monitoring of electronic devices place.

The following sections discuss the proposal of a scalable automation system (section 2) will be described and results are presented in the third section and the conclusions and future work in section four of this work.

\section{Proposal Scalable Automation System}

The system developed consists of four applications:

1. Application of Raspberry: Its purpose is reading the different sensors connected via XBee modules and control actuators in the rooms, in addition to constantly communicate the client application to the server status variables of patients and rooms via web services.

The patient census variables are:

- Body temperature. The patient's temperature is acquired constantly.

- Heart rate. The patient's heart rate is measured constantly.

- Help button. The patient can activate the help button when you require personalized medical care.

In addition an alert notification will be sent if the patient acquired variables are not within the parameters established in the patient module; this alert will notify the medical staff and the head of floor area.

2. Application Administrator: This application is responsible for managing the system; such as add, modify, and delete:

- Users.

- Dispositives.

- Areas.

- Rooms.

- Modules patient.

- Modules room.

3. Client Application: This application will monitor the variables temperature and heart rate of patients in an area; besides having the ability to change the status of 
the devices associated with rooms in the area; likewise provide a tool to add, view and edit notes on a patient's overall purpose.

4. Configuration Application: This application is used by the administrator to modify the individual configuration of modules and patient room.

\section{Users}

As users of the system, four users are defined:

1. Patient: The patient may use a wired control or a mobile application for managing variables within their environment (room) and notify any problems to medical personnel through a help button, each patient will an associated module containing a unique ID in order to identify at all times in addition to census temperature and heart rate.

2. Medical Staff: The medical staff can view the information of the variables (temperature, heart rate and alarm status and emergency aid) of all patients being acquired by its respective module; You can also add and view notes general purpose patients can define whether the note is visible or not for them, in order to be able to deal personally sensitive issues; eg diagnosis of a terminal illness. This will be done from the mobile application and access the system through a username and password.

3. Head of Floor: The floor manager will have a username and password to access the system; you can add new patients also display the information of patients assigned in your area as well as view the notes of these patients and may also set up communication with the server.

4. Administrator: The administrator is the only user who can configure the system, and manage users and system components, adding, modifying and deleting, areas, rooms, beds, Devices and Users.

\section{System Architecture}

The system architecture, Figure 1 shows the basic layout of the sis-theme, which is composed of five parts:

1. Server: This is where it is hosted database connection services both users, areas, devices and patients.

2. Client: they are mobile devices that communicate with the system via the local area network; have an application which allows you to control an environment which is accessed from a code room or in the case of medical personnel log so that they can perform various activities such as monitoring patient variables.

3. Raspberry: Stores the core application system, which is responsible for obtaining the information from the various modules connected to the system at that time and synchronize them with the database using web services. This application is managed by the floor manager.

4. Module Patient: A controller card where the sensors are connected to obtain the patient's vital signs as well as the help button if the patient needs personalized care.

5. Control Module Room: This controller card contains the environmental control modules which allow the handling of actuators and temperature sensors, presence, smoke and humidity of the room. 


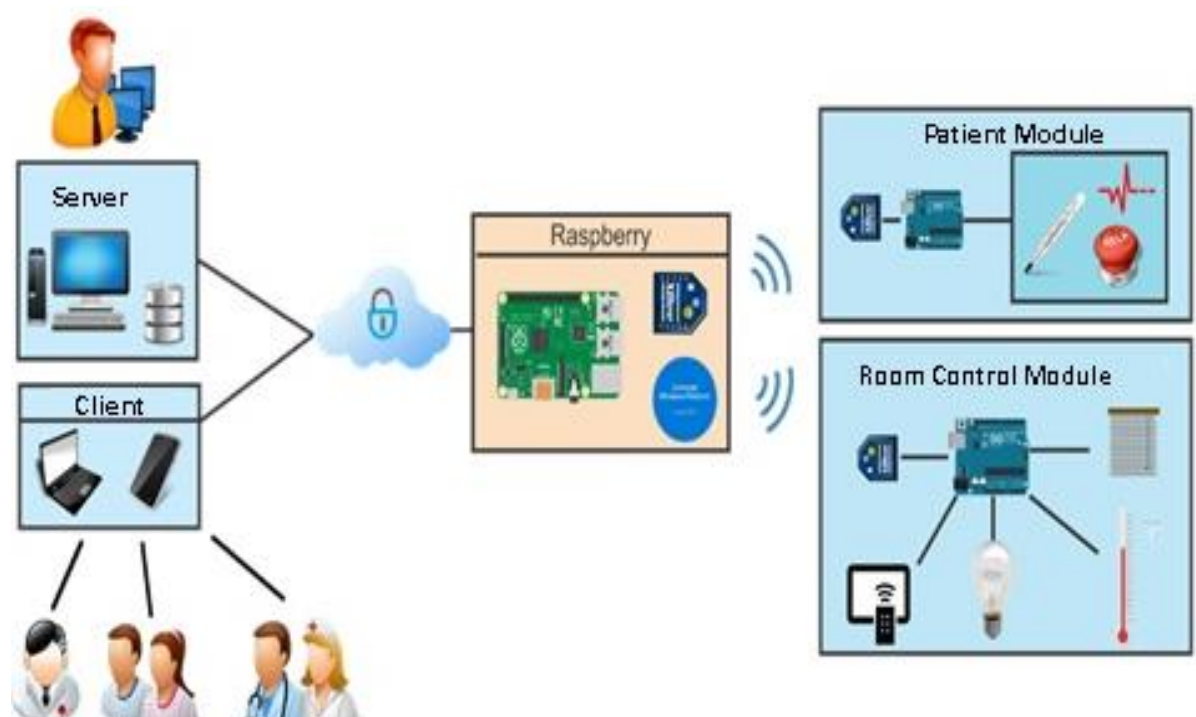

Fig. 1. System architecture.

\section{Patient module}

The patient module is responsible for notifying the state of the variables thereof, and if required assistance or have any kind of difficulty. It consists of an Arduino Nano, one XBee-S2 indicators in LED module, an LCD display, a key button for medical staff and a detachable sensor module. The module has a unique ID.

The LCD display allows us to monitor the patient's condition locally even when there is no network available ZigBee and if you have temperature or high pulse or low. LEDs indicate the same way if the patient needs help, or reading any of its variables is outside the set range. Only alerts can be disabled using button key carrying the doctor, in order to prevent the patient clear the alert.

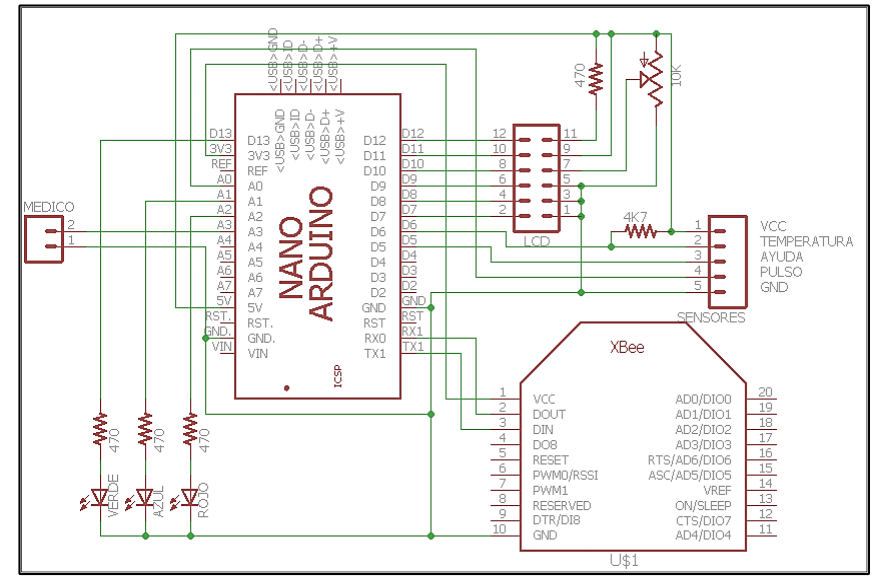

Fig. 2. Schematic module patient. 


\section{Room module}

The room module is in charge of census variables Room and manage the status of the actuators. It is designed in a modular way allowing you to connect and disconnect 2 modules using 2 digital outputs, one of which supports PWM on both channels and 4 digital output modules, 2 of which support PWM. This modularity and the possibility to configure the Arduino make the room module can control subsequent designs requiring minimal or no changes in the minimum hardware and software changes.

This module includes a base for connecting the XBee and a voltage regulator which allows regulated output of $5 \mathrm{~V}$ up to $1 \mathrm{~A}$, just as you have a Molex 20516 -pin connector which allows the connection of a sensor card and PRT-00116 connector 6way for connecting a wireless keyboard which allows use of the module even if there is a network problem.

The census module of the room is a module that integrates the use of temperature sensors, relative humidity, presence and smoke, in addition to handling an actuator for Buzzer or LED depending on the type of alarm required.

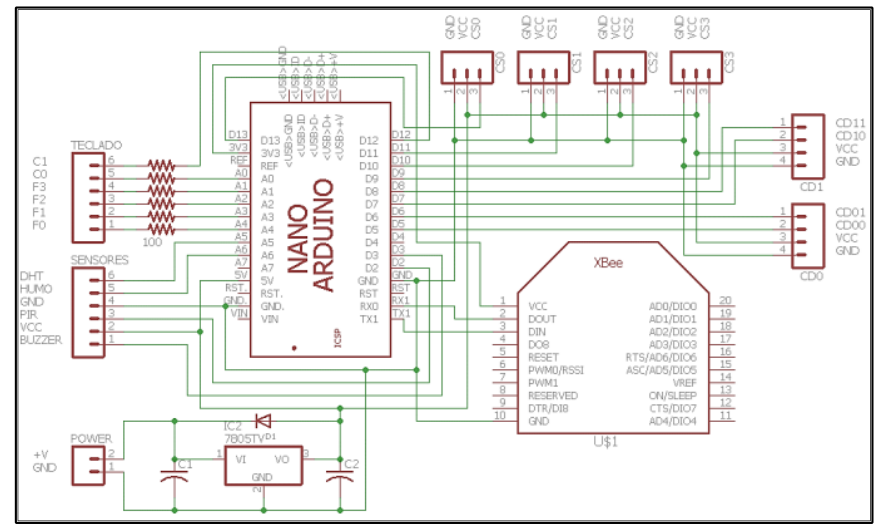

Fig. 3. Schematic room module.

\section{Communication frame}

For communication between applications and modules or patient room, a frame composed with the following elements were designed:

- ID Arduino,

- ID Area,

- Patient indicator plot,

- Patient Temperature,

- Heart rate,

- Alarm Help,

- Emergency alarm,

- Frame Indicator Room,

- Room temperature,

- Room humidity,

- Time since last reading, 
- $\quad$ Alert of smoking,

- State of the actuators.

As we can see, in this plot we integrate all acquired values, both the patient and the room and the status of devices connected to the room. However it is important to note that all fields are sent without differentiating the module that sends this to standardize the size of the plot only that frame indicators defined what information will be used for each.

\section{Web Services}

A web server implemented in language services was used python using the platform django rest framework, these services ensure the consistency of all operations performed on our database and maintain coordinated our "Raspberry", "App Mobile" applications and "Administrator" to work together in our system.

The answers and requests to the server are made using JSON objects containing the requested information and / or issued later to be serialized or deserialized to obtain the information. These objects use HMACs to validate their authenticity.

Management services (high, low and changes) were performed and frequent consultation services such as the status of devices in the room or the temperature and heart rate of patients.

\section{$3 \quad$ Results}

The resulting system was applied in areas of the School of Computing of the IPN; with what was observed system functionality realized in a real room.

By implementing this system, we note that it has a delay of about 1 to 2 seconds to send signals on / off devices; but the system meets the functionality to what was proposed, in addition to the synchronization between the application of mobile application Raspberry and these in turn with the room modules and patient achieved.

On the other hand, from the census temperature and heart rate of several people through our system, and comparing with other commercial sensors; we noted that temperature has a difference of \pm 0.1 degrees between the two sensors, and heart rate as to variations in the census depend on the position in which the sensor is placed in the patient.

\section{Conclusions}

Unlike home automation systems for existing hospitals, it was possible to integrate part of device control room and monitoring variables heart rate and temperature of the patient in a single system that alerts the medical staff when the patient variables are outside established ranges, plus alert if any smoke in one of the rooms the area so that they can notice of a fire and make evacuate the building to avoid accidents.

On the other hand, the aim of developing a system using Raspberry Pi microcomputer, which allows patients to monitor and allows the user to control the devices in a controlled physical environment that adapts to user needs was achieved. 
This system is a scalable system because it is designed so that if the place where this or will be installed the system grows in relation to users and areas, only simply add more a microcomputer in the event that a new area is added, or add a new patient module if you need to monitor a person more and add a new room module if only increase the rooms that are to be controlled.

Among the features of the system developed we find that it is an adaptable system, because not only can be applied to hospitals or care for the elderly; with small changes you can adapt the system to be applied in a room or home school.

Finally we can say that is a system fault tolerant because even though if for some reason fails to operate the mobile application, the application of Raspberry or server the user can continue to control the devices in the room using the keyboard or you can view the status of patient variables using the LCD located on the module that each patient brings.

Acknowledgements. The authors of this paper thank Instituto Politécnico Nacional (IPN), ESCOM, CIC, COFAA, SIP support and facilities for the development of this work.

\section{References}

1. Dutta, S., De Meyer, A.: Building Assets in Real Time and in Virtual Space - Second Part. Denmark: Knowledge Management INSEAD (2013)

2. Kalz, M.: Building Eclectic Personal Learning Landscapes with Open Source Tools. In: Conference proceedings for the Open Source for Education in Europe, Research \& Practise conference. Heerlen: Open University of the Netherlands (2014)

3. Anon: Available at: http://akizukidenshi.com/download/ds/aosong/DHT11.pdf (2015)

4. Arduino: Arduino-ArduinoBoardNano. Available at: https://www.arduino.cc/en/Main /ArduinoBoardNano (2015)

5. ElProCus: Electronic Projects for Engineering Students, What is ZigBee Technology, Architecture and its Applications? Available at: http://www.elprocus.com/what-is-zigbeetechnology-architecture-and-its-applications (2014)

6. Mitsucontrol: Ahorro de energía. Available at: http://www.mitsucontrol.com.ar (2015)

7. Item Raspberry Pi: Raspberry Pi - Teach, Learn, and Make with Raspberry Pi. Available at: https://www.raspberrypi.org (2015)

8. Raspberryshop: Hardware Raspberry Pi. Available at: http://www.raspberryshop.es /hardware-raspberry-pi.php (2015)

9. 4pcb: PCB Printed Circuit Board File Creation Calculator, Advanced Circuits. Available at: http://www.4pcb.com/trace-width-calculator.html (2015) 